\title{
A Syndromic Approach to Emergency Department Surveillance for Skin and Soft Tissue Infections
}

\author{
Larissa May*1, Marcus Rennick², Leah Gustafson ${ }^{1}$ and Julia Gunn ${ }^{2}$ \\ ${ }^{1}$ Emergency Medicine, The George Washington University, Washington, DC, USA; 'B Boston Public Health Commission, Boston, MA, \\ USA
}

\section{Objective}

We sought to describe the epidemiology of emergency department (ED) visits for skin and soft tissue infections (SSTI) in an urban area with diverse neighborhood populations using syndromic surveillance system data for the time period from 2007-2011. Our aims were threefold: to demonstrate a proof of concept using syndromic surveillance for SSTI surveillance in the absence of laboratory data, to estimate the burden of ED visits associated with SSTI, and to determine potential geographic "hotspots" for these infections.

\section{Introduction}

The incidence of and hospitalizations for SSTI have steadily increased over the last decade in the United States, primarily due to the emergence and spread of community acquired Methicillin resistant Staphylococcus aureus (CA-MRSA). The ED is a common site for SSTI treatment and serves populations not captured by traditional surveillance, including the homeless and uninsured. The use of near real-time syndromic surveillance within the ED to detect unusual activity for further public health investigation has been used to augment traditional infectious disease surveillance. However, the use of this approach for monitoring local epidemiologic trends in SSTI presentation where laboratory data are not available, has not previously been described.

\section{Methods}

We sought to describe the epidemiology of ED visits for SSTI in an urban area with diverse neighborhood populations using the Boston Public Health Commission's (BPHC) Syndromic Surveillance System (BSynSS) data for a five year time period (2007 through 2011). SSTI related visits were defined by either chief complaints with SSTI associated words (abscess, cellulitis) or final diagnosis International Classification of Diseases (ICD-9 CM) codes for SSTIs. SSTI related visits were de-duplicated using demographics and visit identifiers and then stratified by age group, gender, race, and neighborhood of residence defined by ZIP code. Each of Boston's 15 neighborhoods has a unique demographic profile with distinct differences in race, socioeconomic status, and age. Finally, we examined trends in characteristics of potential "hotspots" of neighborhood clustering for SSTIs in EDs.

\section{Results}

Using our SSTI syndrome definition, we estimated unique SSTI visits represented $3.29 \%(n=45,252)$ of all visits within Boston's ten EDs during the study period with a seasonal pattern peaking during the summer months (July through September). The majority of SSTI visits $(54 \%)$ were among patients 18 to 44 years old, which is consistent with the age distribution of the Boston population. However, a disproportionate number of SSTI visits (43\%) were among Black patients when compared to both the overall Boston population $(22 \%$ Black) and to the racial distribution of all ED visits (39\% Black). The five-year average rate of SSTI visits for Black patients (281.2 per $10,000$ population) was significantly greater at 2.8 times [CI $2.7,3.0]$ than the rate for White patients ( 99.0 per 10,000 population). Geographic neighborhood distribution of SSTI visits ranged from a low of $2.69 \%$ to a high of $4.11 \%$ of all neighborhood-specific ED visits. Disposition data are available for 2010 and 2011 only and show that $24 \%$ and $23 \%$ of patients in 2010 and 2011, respectively, were admitted for their SSTI.

\section{Conclusions}

Our study results suggest that syndromic surveillance data can be used to track the burden and patterns of SSTI in an urban population, including disease severity through the use of disposition data. Furthermore, syndromic surveillance can provide information on the local epidemiology of SSTI, including data related to health inequalities. The burden of SSTIs should be compared to overall ED use for a specific population to control for biases in health care seeking behaviors and choice of provider type. A local syndromic surveillance system has the potential to provide public health authorities and ED clinicians near real-time monitoring of trends in severity and demographic risk factors, and may provide an alternative to tracking the severity of illness where no laboratory data are readily available.

\section{Keywords}

syndromic surveillance; epidemiology; skin and soft tissue infections; racial disparities

\footnotetext{
*Larissa May

E-mail: larissa.may@gmail.com
} 